\title{
VULNERABILIDAD FÍSICA DE CUBIERTAS DE EDIFICACIONES DE USO DE OCUPACIÓN NORMAL ANTE CAÍDAS DE CENIZA EN LA ZONA DE INFLUENCIA DEL VOLCÁN GALERAS
}

\author{
Roberto Armando Torres-Corredor ${ }^{1 *}$; Patricia Ponce-Villarreal ${ }^{1}$; Diego Mauricio Gómez-Martínez ${ }^{1}$
}

DOI: http://dx.doi.org/10.18273/revbol.v39n2-2017005 @c) (1)

Forma de citar: Torres-Corredor, R.A., Ponce-Villarreal, P., y Gómez-Martínez, D.M. 2017. Vulnerabilidad física de cubiertas de edificaciones de uso de ocupación normal ante caídas de ceniza en la zona de influencia del volcán Galeras. Boletín de Geología, 39(2): 67-82.

\section{RESUMEN}

El enfoque principal de esta investigación es un método para evaluación de la vulnerabilidad física de cubiertas de edificaciones por sobrecarga ante caída de ceniza, por erupciones del volcán Galeras. Para conocer la posible afectación de cubiertas y edificaciones, se establecen relaciones de daño para tipos de cubiertas con base en su tipología, distancias entre apoyos y estado. La evaluación de vulnerabilidad permite estimar el grado de daño probable de la cubierta y edificación. La metodología parte de caracterizar la amenaza para establecer el patrón de intensidad para analizar la resistencia del elemento expuesto, que para ceniza, es la carga transferida por un espesor acumulado sobre la cubierta. Adicionalmente, se realizó un inventario de elementos expuestos, definiendo tipo de cubierta, materiales, configuración geométrica y estado, así como el de la edificación, para luego categorizar en cubiertas tipo, a las cuales se les analiza la resistencia para determinar la máxima solicitación que conduce al fallamiento, evaluando también el impacto sobre toda la edificación. Se estima la probabilidad de daño mediante una función de distribución acumulativa, teniendo en cuenta la varianza de la solicitación límite debido a factores como heterogeneidades de los materiales, dimensiones de elementos, efectos constructivos o distribución de la carga de ceniza de acuerdo a la trayectoria del viento, al estilo de cubierta, así como la humedad presente en la ceniza. La evaluación de vulnerabilidad en la zona de Galeras muestra que aunque las cubiertas livianas y moderadas son más vulnerables a cargas de cenizas, el efecto de afectación global en la edificación es menor, contrario a cubiertas pesadas y losas. Este estudio formula consideraciones en diseño de cubiertas de edificaciones ubicadas en zonas aledañas a volcanes activos, que podrían ser base para la creación de una norma de diseño y construcción para cubiertas resistentes ante sobrecargas de ceniza.

Palabras clave: Vulnerabilidad, cubiertas de edificaciones, caída de ceniza, volcán Galeras.

\section{PHYSICAL VULNERABILITY OF BUILDING ROOFS OF NORMAL OCCUPANCY USE FACING ASHFALL IN THE GALERAS VOLCANO INFLUENCE ZONE}

\begin{abstract}
The main focus of this research is a method for assessing the physical vulnerability of building roofs due to overloading of ashfall by eruptions of Galeras volcano. In order to know the possible affectation of roofs and buildings, damage relationships are established for types of roofs based on their typology, distances between supports and state. The vulnerability assessment allows estimating the likely degree of damage of the roof and building. The methodology starts from characterizing the hazard to establish the intensity pattern to analyze the resistance of the exposed element, which for ash, is the load transferred by a thickness accumulated on the roof. Additionally, an inventory of exposed elements was made, defining type of roofs, materials, geometric configuration and state, as well as of the building, which allow to categorize in roof types. Then the resistance is analyzed in order to determine the maximum stress that leads to failure, evaluating also, the impact on the entire building. The probability of damage is estimated using a cumulative distribution function, taking into account the variance of the boundary stress due to factors such as material heterogeneities, element dimensions, constructive effects or ash load distribution according to the wind path, roof style, as well as the moisture present in the ash. The vulnerability assessment in Galeras zone, shows that although light and moderate roofs are more vulnerable to ash loads, the effect of overall damage to the building is less, as opposed to heavy roofs and slabs. This study formulates considerations in the design of building roofs located in adjacent areas to active volcanoes, which could be the basis for the creation of a standard for design and construction of overload ash-resistant roofs.
\end{abstract}

Keywords: Vulnerability, building roofs, ashfall, Galeras volcano.

\footnotetext{
${ }^{1}$ Servicio Geológico Colombiano. Pasto, Nariño, Colombia. (*)rtorres@sgc.gov.co, pponce@sgc.gov.co,dgomez@sgc.gov.co
} 


\section{INTRODUCCIÓN}

Una amplia gama de amenazas están relacionadas directamente con la actividad volcánica y durante tiempos históricos todas han causado pérdidas de vidas humanas, de propiedades y de recursos. El Galeras es considerado uno de los volcanes con actividad eruptiva más recurrente en Colombia y dentro de su actividad ha producido erupciones de tipo vulcaniano con unos 60 registros eruptivos entre 1500 y 1936 y 21 erupciones explosivas desde 1989, tiempo desde que se dio inicio a su vigilancia continua por parte del entonces INGEOMINAS, hoy Servicio Geológico Colombiano (SGC). El Galeras que cuenta con importantes asentamientos humanos en sus laderas, incluyendo la ciudad de San Juan de Pasto (capital del Departamento de Nariño), en los años noventa fue escogido por la International Association of Volcanology and Chemistry of the Earth's interior (IAVCEI) como uno de los volcanes de la Década como parte del programa de las Naciones Unidas para la Reducción de Desastres Naturales. La actividad de Galeras se ha caracterizado por erupciones acompañadas por caídas de cenizas. Algunas de estas caídas han durado horas o días cubriendo áreas relativamente amplias alrededor de la zona de influencia del volcán. Como parte de la Gestión del Riesgo, el SGC ha llevado a cabo estudios de evaluación de la vulnerabilidad física ante caída de cenizas en la zona de influencia del volcán. Este estudio se enfoca en una metodología que se ha utilizado en la evaluación de la vulnerabilidad ante la caída de cenizas por eventos eruptivos en Galeras teniendo en cuenta el tipo de estructuras expuestas y haciendo hincapié en los techos y las edificaciones. Es indispensable contar con edificaciones con capacidad para resistir los efectos probables de una erupción, incluyendo la acumulación de ceniza a fin de reducir la urgencia de una posible evacuación o facilitar la reocupación y la recuperación posterior a la erupción.

\section{GENERALIDADES}

El volcán Galeras es un estrato-volcán que alcanza una altura máxima de $4276 \mathrm{msnm}$ y se localiza en el límite oriental de la depresión interandina CaucaPatía en el Departamento de Nariño a $1^{\circ} 13,26^{\prime} \mathrm{N}$ y $77^{\circ} 21,54^{\prime} \mathrm{W}$. Su cráter activo se ubica a $9 \mathrm{~km}$ al W de San Juan de Pasto, capital del Departamento de Nariño que cuenta con 445500 habitantes según proyección DANE para 2016. El edificio volcánico de Galeras, con una edad aproximada de 4500 años, se caracteriza por la presencia de depósitos de flujos de lavas andesíticas, flujos piroclásticos y depósitos de caída, levantados dentro de una serie de complejos volcánicos más antiguos. Su actividad principalmente se ha caracterizado por la ocurrencia de emisiones de gas y ceniza, flujos de lava andesítica y erupciones vulcanianas que han ocasionado depósitos de caída y de flujos piroclásticos (Calvache, 1990).

INGEOMINAS (1997) publica la tercera versión del Mapa de Amenaza Volcánica de Galeras demarcando tres zonas de amenaza (FIGURA 1). La zona de amenaza alta corresponde a un sector afectado con una probabilidad mayor al $20 \%$ de que ocurran eventos volcánicos con la máxima severidad (cinco) asociados con flujos piroclásticos donde se esperaría que no haya sobrevivientes y las propiedades sean destruidas a lo largo de su trayectoria; adicionalmente, esta zona sería afectada por flujos de lava, caídas piroclásticas, flujos de lodo, proyectiles balísticos, ondas de presión acústica (ondas de choque) y altas concentraciones de gases en inmediaciones del cono activo. Erupciones de tamaño similar a las ocurridas en 1989, 1992 y 1993 generarían depósitos de caída piroclástica con espesores de orden métrico en las proximidades del cráter, y de orden centimétrico y milimétrico a distancias mayores a $1 \mathrm{~km}$ y en el caso de erupciones como las de los últimos 5000 años se esperaría que se generen caídas piroclásticas con espesores superiores a $30 \mathrm{~cm}$ en distancias menores a $5 \mathrm{~km}$ del cráter activo. La zona de amenaza media corresponde también a los fenómenos descritos en la zona de amenaza alta, pero dados por ocurrencia de erupciones más grandes, con una probabilidad entre el 10 y $20 \%$ con severidades entre 3 y 5 , y donde se contemplan los efectos de la onda de calor asociada a los flujos piroclásticos. La zona de amenaza baja está definida por zonas que estarían afectadas por una probabilidad inferior al $10 \%$ con severidad igual o mayor a 2; enmarcada principalmente por las tendencias de depósitos de caídas piroclásticas que incluyen la caída de ceniza (partículas con tamaños no superiores a los $2 \mathrm{~mm}$ ) y por la onda de presión acústica.

Los procesos eruptivos más comunes en Galeras conllevan a las caídas de ceniza. El magma es expulsado en forma de fragmentos líquidos y sólidos, que son eyectados hacia arriba desde el cráter para formar una columna o nube de material transportado por el aire, del cual las partículas más finas son arrastradas por el viento. Los fragmentos de mayores tamaños caen rápidamente en el área más cercana al volcán y los más pequeños a mayores distancias. Los efectos de las caídas de cenizas varían ampliamente, dependiendo del volumen de material expulsado y la duración o intensidad de la erupción, vientos y condiciones de 
humedad. En las zonas próximas al volcán, las cenizas pueden cubrir terrenos dedicados a la agricultura, afectando los cultivos.

La acumulación de ceniza puede producir enterramiento de estructuras, empuje lateral de muros, sobrepeso y desplome en los techos de las viviendas. Es importante tener en cuenta que según el tamaño de la ceniza y su grado de hidratación, puede penetrar en estructuras y causar daños a materiales y equipos dentro de las edificaciones. Adicionalmente, las superficies exteriores de las viviendas, en especial aquellas que están expuestas a la lluvia, también pueden sufrir por efecto de la ceniza contaminada con compuestos ácidos emanados del volcán y que reaccionan con el agua. El polvo en el aire puede conducir a efectos nocivos en las personas y animales (problemas respiratorios, irritación en las mucosas, abrasión en dentaduras, etc.), causar daños en acueductos, redes eléctricas y telefónicas como también afectar la aeronavegación. De acuerdo con el mapa de amenaza volcánica de Galeras (INGEOMINAS, 1997), la línea que conforma el borde externo del óvalo del mapa de amenaza, puede acumular hasta 1,0 mm de ceniza y conforme a los registros geológicos en el interior del óvalo, los espesores han sido variables del orden de centímetros y en general menores a los $40 \mathrm{~cm}$.

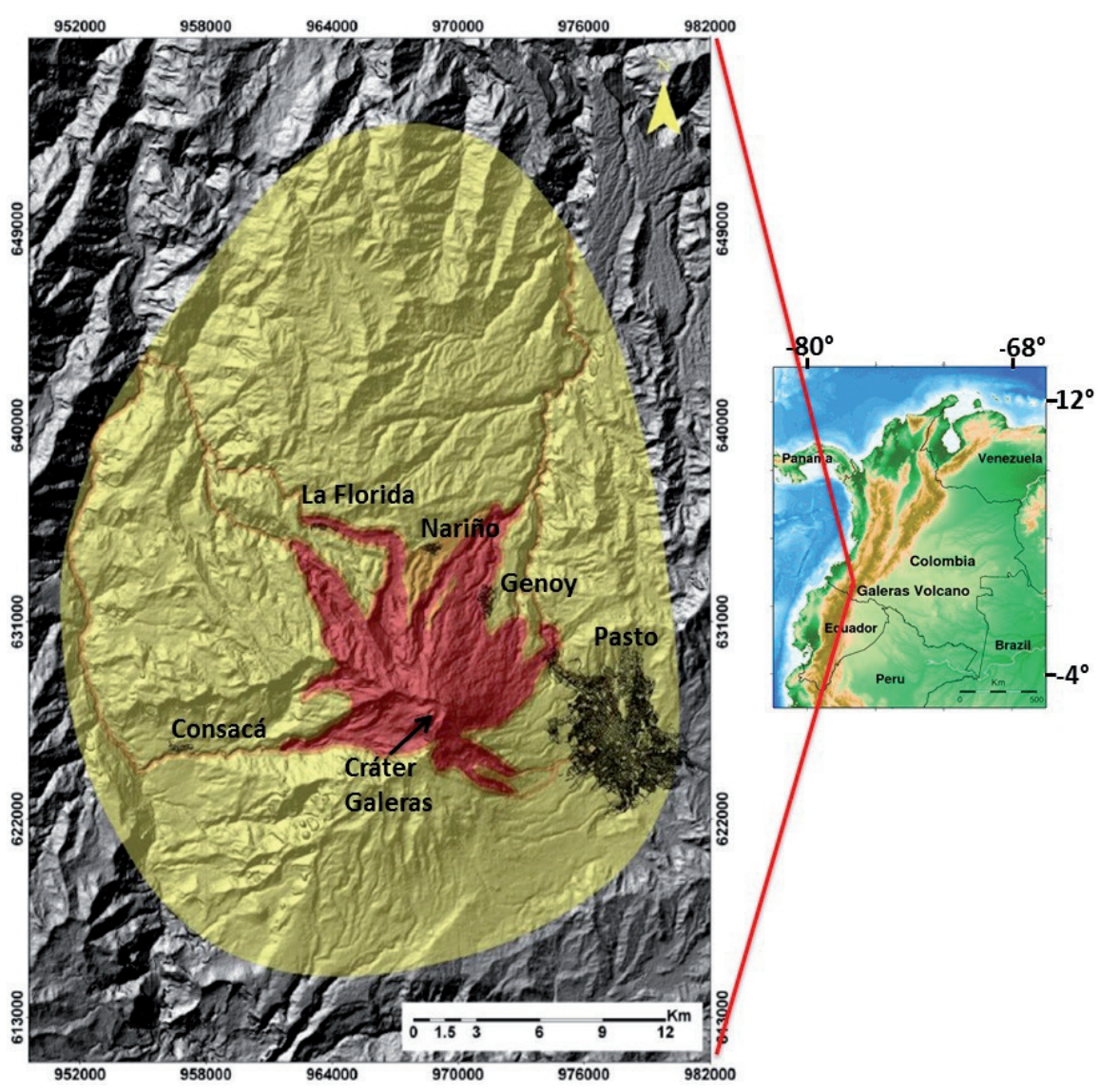

FIGURA 1. Mapa de localización del volcán Galeras y de Amenaza volcánica de la tercera versión. El recuadro ampliado donde se muestra en colores las zonas de amenaza está en coordenadas planas de Gauss origen Buenaventura. El color rojo corresponde a la amenaza alta, el anaranjado a la amenaza media y el amarillo a la amenaza baja. La flecha apunta al cráter activo principal del volcán y en el mapa se destaca la ciudad de Pasto y algunos centros poblados.

\section{ANTECEDENTES}

INGEOMINAS (1998) realizó un trabajo sobre vulnerabilidad de viviendas de ocupación normal en el área de Galeras con base en estudios analíticos de estimaciones de la respuesta y resistencia de los elementos a cargas de solicitación con un enfoque estático en función de los tipos de materiales, configuraciones geométricas (inclinación de cubierta, longitud entre apoyos y correas, y secciones transversales de elementos entre otros), ensambles-conexiones, la trasferencia de las cargas a los diferentes elementos de la cubierta, continuidad de los elementos, estado y edad de las cubiertas entre otros aspectos. 
Entre 2001 y 2006, INGEOMINAS implementó un estudio de cargas de colapso de techos con base en los resultados de los estudios de techos en la región de Galeras y las experiencias de daños observados en Filipinas con la erupción del Pinatubo en 1991 y en la ciudad de Rabaul en Papua Nueva Guinea por la erupción del Tavurvur en 1994 (Torres and Cárdenas, 2006); este estudio condujo a mostrar el colapso de techos para diferentes escenarios de espesores de cenizas (FIGURA 2).

$3 \mathrm{~cm}$ de espesor de ceniza

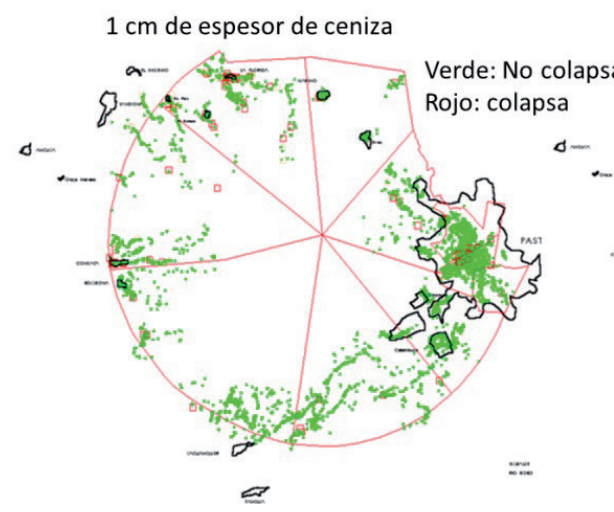

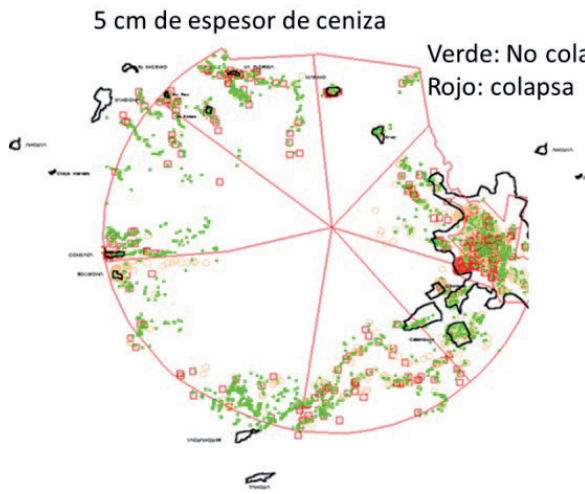

$10 \mathrm{~cm}$ de espesor de ceniza

\section{3}

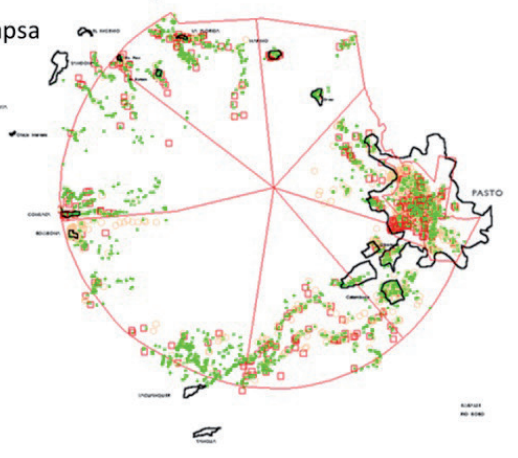

$10 \mathrm{~cm}$ de espesor de ceniza

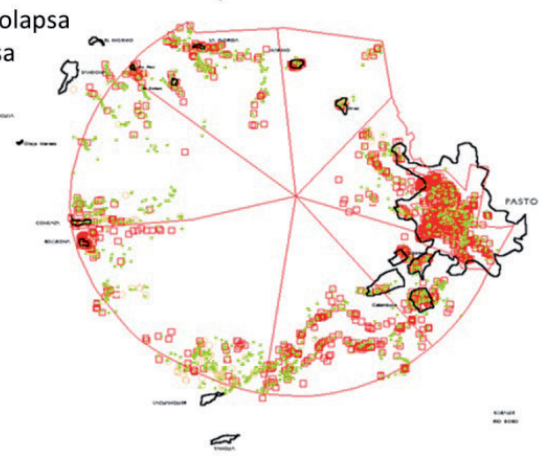

$20 \mathrm{~cm}$ de espesor de ceniza

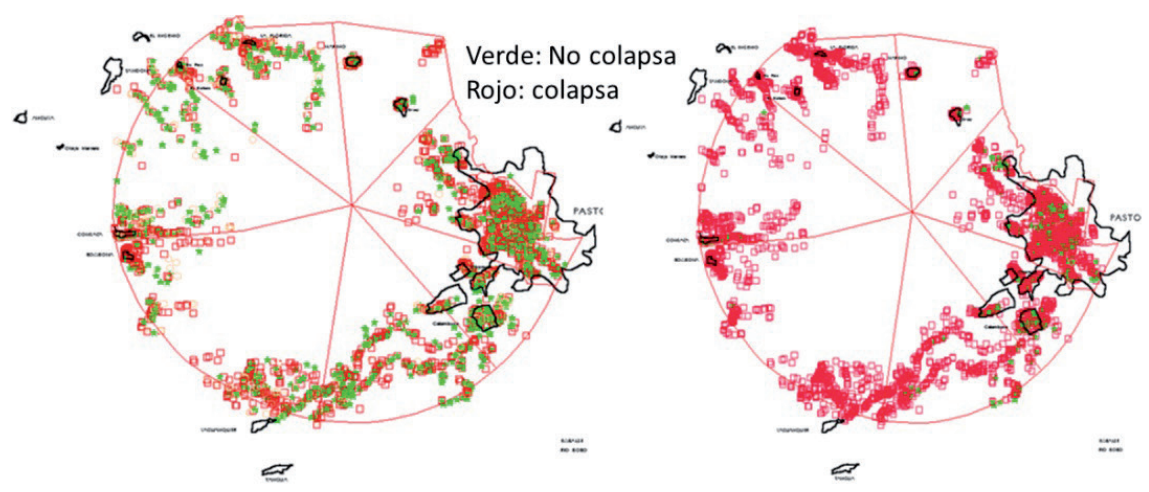

FIGURA 2. Distribución de cubiertas de viviendas en el área de Galeras que colapsan con diferentes espesores de depósitos de ceniza húmeda (Torres and Cárdenas, 2006).

CORPOSSO (2009), llevó a cabo un estudio de vulnerabilidad de cubiertas ante caída de cenizas volcánicas como parte del Estudio de Vulnerabilidad física y funcional a fenómenos volcánicos en el área de influencia del volcán Galeras, estableciendo funciones de probabilidad de daño, tomando en consideración los estudios de López (1997), INGEOMINAS (1998), Spence et al. (2005) y Zuccaro et al. (2008).
En 2012, teniendo en cuenta la experiencia del SGC en el contexto del fenómeno volcánico, la evaluación de la amenaza volcánica y de la vulnerabilidad física de algunos elementos expuestos, así como por la disponibilidad de información colectada durante el ejercicio del estudio y vigilancia de los volcanes activos del país, entra a hacer parte de un Proyecto de Asistencia Técnica (TAP) para una Evaluación Probabilista del 
Riesgo Volcánico (EPR) tomando como área piloto el volcán Galeras (SGC-UNGRD, 2012) y la utilización de la plataforma y herramienta de análisis CAPRA (ERNAL, 2009), obteniendo la estimación de funciones de vulnerabilidad con base en los productos arrojados por el volcán, especialmente caídas de ceniza (Torres et al., 2012). Dorado (2013) desarrolla la evaluación de la vulnerabilidad de edificaciones ante caída de cenizas en la cabecera urbana del Municipio de Consacá Departamento de Nariño con la asesoría del SGC.

\section{CONTEXTO TEÓRICO Y METODOLOGÍA}

Como punto de partida, se considera como vulnerabilidad física al grado de pérdida o de daño de un elemento o de un conjunto de elementos bajo riesgo ante la acción de un fenómeno natural de cierta intensidad (magnitud) y se puede calificar desde no daño, $0(0 \%$ de daño) a daño o destrucción total, 1 (100\% de daño). A fin de poder establecer la susceptibilidad de un elemento bajo riesgo de sufrir daño ante un fenómeno de amenaza, la vulnerabilidad física puede establecerse funcionalmente como la relación entre el nivel de exposición del elemento tomado como la intensidad prevista o esperada de una amenaza específica en el sitio del elemento, y la resistencia como la capacidad de carga límite que el elemento ofrece ante la acción del fenómeno amenazante y que determina su funcionalidad (Díaz-Granados, 1994).

Con el propósito de obtener el grado de daño esperado de un elemento bajo riesgo es posible optar por determinar la relación del costo de reparación al costo de reemplazamiento del elemento como una función de la intensidad de la amenaza (UNDRO, 1991). La metodología para la evaluación de la vulnerabilidad parte de la caracterización de la amenaza en consideración para poder establecer el patrón de intensidad que se tendrá como referente para el análisis de resistencia del elemento expuesto, que en el caso de la ceniza, es la carga transferida por un cierto espesor de material acumulado sobre la cubierta; por otra parte, se establece un inventario de elementos expuestos en la zona objeto de estudio que para el caso son las cubiertas con la edificación. A las cubiertas se les define el tipo de estructura, materiales, configuración geométrica y la condición de su estado para hacer una categorización que permita tener unas cubiertas tipo. Para cada cubierta tipo se procede a realizar un análisis de resistencia a fin de determinar la máxima solicitación que conduce a su fallamiento y se procede a evaluar también el impacto sobre toda la edificación.
Establecidas las solicitaciones límite que conducen a la falla se procede a estimar la probabilidad de daño mediante una función de distribución acumulativa teniendo en cuenta la varianza de la solicitación límite debido a las heterogeneidades propias de los materiales, dimensiones de los elementos y efectos constructivos o a la distribución de la carga de ceniza de acuerdo a la trayectoria del viento y al estilo de la cubierta así como la humedad presente en la ceniza entre otros posibles. La FIGURA 3 esquematiza mediante un flujograma el proceso metodológico para evaluación de la vulnerabilidad física de elementos expuestos o bajo riesgo.

En primera instancia el análisis de vulnerabilidad física solamente concierne a lo que tiene que ver con el impacto en la calidad de la obra de construcción, en sus elementos o materiales en cuanto a una disminución significativa de su resistencia; no obstante, también el concepto se ha extendido a la consideración de la pérdida de funcionalidad. La intensidad de las caídas de ceniza sobre elementos de cubiertas se puede establecer mediante las cargas ocasionadas por la ceniza depositada o su equivalente espesor.

El mecanismo de falla se hace mediante la imposición de un exceso de carga sobre la cubierta hasta alcanzar el valor de colapso o de desplome considerando cada uno de los elementos que hacen parte de la estructura de cubierta y los condicionantes relacionados con la aplicación de la carga, el estado de la estructura, su configuración geométrica, los materiales, el dimensionamiento y el ensamble de los elementos que la conforman según su tipología (Torres, 2001).

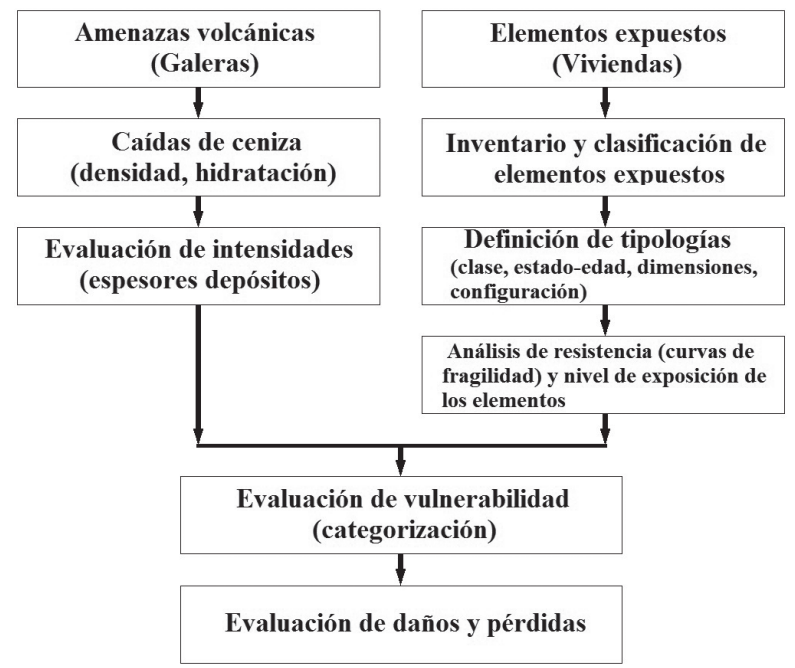

FIGURA 3. Flujograma de la metodología para evaluación de la vulnerabilidad física (Modificado de Torres, 2001). 
Con base en las cargas límite de colapso por unidad de área $Q_{\text {mean }}$, estimadas por López(1997) y Torres (2001), se procede a calcular la probabilidad de colapso $p$ (colapso). Según Spence et al. (2005), la probabilidad de colapso se puede determinar mediante una distribución normal acumulativa $\Phi$ con valor medio $Q_{\text {mean }}$ y desviación estándar $Q_{\text {dev }}$; donde $Q_{\text {mean }}$ corresponde al valor medio de la carga límite de la cubierta por unidad de área, y $Q_{d e v}$ es la desviación estándar de la carga límite sobre la cubierta por unidad de área.

$$
p(\text { colapso })=\Phi\left(Q_{\text {mean }}, Q_{\text {dev }}\right)
$$

La función de densidad de probabilidad está dada por:

$$
P\left(Q_{i}\right)=\varphi_{Q_{\text {mean }}, Q_{d e v}^{2}}(Q)=\frac{1}{\sqrt{2 \pi} Q_{\text {dev }}} e^{\left(\frac{\left(\frac{\left(-Q_{\text {mean }}\right)^{2}}{2 Q_{\text {dev }}^{2}}\right)}{}\right.}
$$

y la función de distribución acumulada correspondiente es:

$$
\Phi\left(Q_{\text {mean }}, Q_{\text {dev }}\right)=\sum_{i=1}^{N} P\left(Q_{i}\right)
$$

La vulnerabilidad se puede expresar mediante funciones que se denotan mediante curvas que relacionan los valores esperados de la Relación Media de Daño (RMD), referidos como $\mathrm{E}\left(\beta \mid \gamma_{i}\right)$, y su desviación estándar con respecto a una medida de las intensidades del evento en consideración $\gamma_{i}$ (Miranda, 1999). La RMD asignada a la cubierta no es igual a la de la edificación completa, entonces se hace necesario establecer cuál es la RMD global a partir de RMD de las cubiertas. El comportamiento global de la edificación puede variar significativamente con respecto al comportamiento de la cubierta. Esta afectación global puede establecerse como la relación del costo de remplazar el elemento o elementos afectados como la cubierta, entrepisos u otros, e inclusive toda la edificación, respecto al costo total de la edificación misma. Este valor podría ser el $100 \%$ si el daño es total para toda la edificación (colapso de todo el edificio), o un porcentaje menor si además de la cubierta se afecta una parte de la edificación.

Para la construcción de las funciones de vulnerabilidad se sigue la metodología empleada en el documento ERN-CAPRA-T1-5 (ERN-AL, 2009), en donde se describe que para un valor dado de intensidad $\gamma_{i}$, el valor esperado de daño $\beta$, se puede determinar con base a la siguiente ecuación (Miranda, 1999; Ordaz, 2000):

$$
E\left(\beta \mid \gamma_{i}\right) D_{\max }=\left(1-e^{\left(\frac{\gamma_{i}}{\gamma^{\prime}}\right)^{\alpha} \ln 0,5}\right) D_{\max }
$$

Donde $\mathrm{E}\left(\beta \mid \gamma_{i}\right)$ es la pérdida esperada para un cierto valor de intensidad $\gamma_{i} ; \gamma_{o}$ y $\alpha$ son parámetros de vulnerabilidad estructural que dependen del sistema estructural y su estado, de modo que $\gamma_{o}$ corresponde al valor de la intensidad para la mitad de pérdida máxima (intensidad en el daño medio) y $\alpha$ es un factor de forma de la curva de la función de distribución de la probabilidad de pérdida. La función de vulnerabilidad empareja los valores de $(0,0),\left(\gamma_{o}, 0,5 \beta_{\max }\right)$ y $\left(\gamma_{u}, \beta_{\max }\right)$ siendo $\gamma_{u}$ la intensidad correspondiente al daño total del elemento.

Para un daño máximo $D_{\max } \leq 1$, la variación de la varianza de la pérdida sigue la expresión del documento ERNCAPRA-T1-5 que refiere a la distribución de probabilidad asignada en el informe ATC-13 (1985) dada por:

$$
V\left(\beta \mid \gamma_{i}\right)=\sigma_{\beta}^{2}\left(\beta \mid \gamma_{i}\right)=Q\left[E\left(\beta \mid \gamma_{i}\right) D_{\max }\right]^{(r-1)}\left[1-E\left(\beta \mid \gamma_{i}\right) D_{\max }\right]^{(s-1)}
$$

Donde $Q, r$ y $s$ son parámetros dados por

$$
s=\frac{r-1}{D_{0}}-r+2
$$

$$
Q=\frac{\sqrt{V_{\max }}}{D_{0}^{r-1}\left(1-D_{0}\right)^{s-1}}=\frac{\sigma_{\max }}{D_{0}^{r-1}\left(1-D_{0}\right)^{s-1}}
$$

Siendo $D_{o}, \sigma_{\max }, r$, parámetros que dependen del tipo de estructura de modo que $D_{o}$ es el nivel de daño respectivo a la desviación máxima $\sigma_{\max }$, y relativamente correspondiente a la intensidad $\gamma_{o}$ (un valor cercano a 0,5 para el daño medio) y $r$ una constante que se tomó como 3,3. En la FIGURA 4 se muestra un ejemplo de función de vulnerabilidad acogiendo las ecuaciones 4 a 7 .

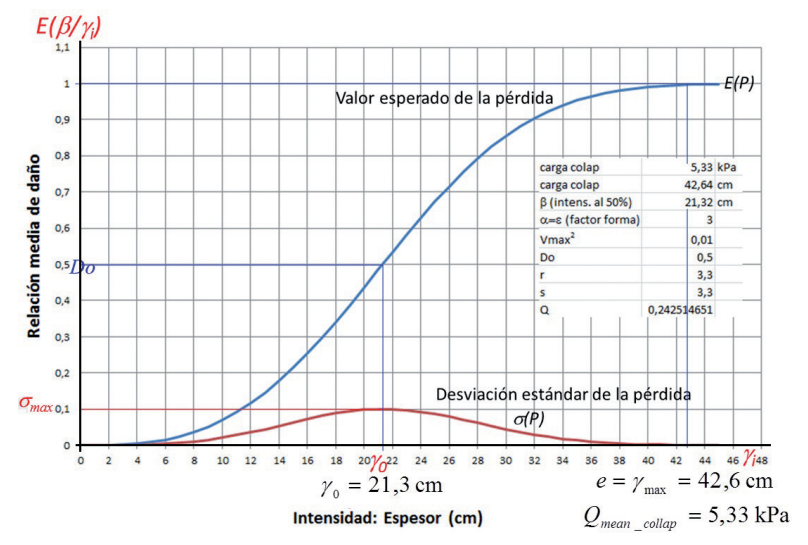

FIGURA 4. Ejemplo de construcción de la función de vulnerabilidad para un elemento con una carga límite de colapso de 5,33 $\mathrm{kPa}$ equivalente a un espesor de ceniza húmeda de 42,6 $\mathrm{cm}$ (peso específico de $1250 \mathrm{kgf} / \mathrm{m}^{3}$ ) con un $D_{\max }=1$.

\section{MECANISMOS DE FALLA POR CAIIDAS DE CENIZA EN CUBIERTAS DE EDIFICACIONES}

En el caso de caída de ceniza, dependiendo de su intensidad, el daño de un elemento expuesto también depende de 
su resistencia. Teniendo en cuenta que los espesores de ceniza esperados por las erupciones de Galeras con base en el Mapa de Amenaza (INGEOMINAS, 1997) son del orden de centímetros y que generalmente no exceden los $40 \mathrm{~cm}$, se descarta el enterramiento de viviendas y los efectos por cargas de empuje lateral a elementos con disposición vertical como puertas, ventanas, etc. La mayor implicación está en la transferencia de cargas verticales a elementos con disposición horizontal o subhorizontal como son los techos o cubiertas de las edificaciones. De acuerdo con la distribución de daños observados en techos con la erupción del Pinatubo en 1991 (Spence et al., 1996), encontraron que, en su orden, las causas de falla y colapso más comunes son: grandes luces (> $5 \mathrm{~m})$, estructuras de cubiertas de madera y grandes voladizos en techos; adicionalmente, los daños fueron mayores en edificaciones no residenciales. El efecto de la pendiente de las cubiertas resultó ser ambiguo, quizás debido a las condiciones de humedad de la ceniza con relación al ángulo de reposo. Spence et al. (2005), también refieren que con $3 \mathrm{kPa}$, para un espesor equivalente de $24,5 \mathrm{~cm}$ de ceniza húmeda, asumiendo un peso específico de 1250 $\mathrm{kgf} / \mathrm{m}^{3}$ (Tilling, 1993) se presentaron daños graves, con una probabilidad de colapso del $33 \%$.

Como se mencionó, INGEOMINAS (1998) realizó un estudio sobre vulnerabilidad de cubiertas de viviendas de ocupación normal en el área del Galeras, con base en un análisis del comportamiento a las solicitaciones de carga de ceniza con aproximación estática, teniendo en cuenta tipo de materiales, configuración geométrica (inclinación de cubierta, longitud entre apoyos y correas, sección de elementos, etc.), la trasferencia de las cargas a los diferentes elementos de la cubierta, estado y edad de las cubiertas entre otros aspectos. En este estudio se establecieron cinco clases de cubiertas, algunas de ellas se ilustran en la FIGURA 5.
Cubierta en teja liviana: techos que tienen como material de cobertura hojas de zinc o aluminio y hasta cartón o similares como marquesinas en marcolita $\mathrm{u}$ otro material acrílico o vidrio. La estructura de cubierta puede ser en madera o metálica.

Cubierta en teja moderada: techos que tienen como material de cobertura tejas de asbesto-cemento o láminas predeterminadas en ciertas cerámicas. La estructura de cubierta puede ser en madera o metálica.

Cubierta en teja pesada: Techos que tienen como material de cobertura tejones o tejas de barro. La estructura que soporta la cubierta usualmente es en madera.

Cubiertas en losa maciza: Placa de concreto armado o bloque macizo acuñado a compresión, la cual va soportada directamente sobre muros o sistemas de pórticos.

Cubiertas en losa aligerada: placa aligerada con nervios en concreto reforzado soportadas directamente sobre muros o sistemas de pórticos.

El estado general de la estructura hace referencia a la calificación del estado de conservación. Para un estado bueno se contempla que no se presenten grietas o fisuras, que exista un buen acople entre los elementos estructurales y en las uniones entre la cubierta con el resto de la edificación, no se observe presencia de humedades o que existe algún tipo de protección o revestimiento contra los efectos del medio ambiente, que no haya presencia de hormigueros u oquedades en el concreto, capas gruesas de óxido en elementos metálicos o que no haya presencia de ataques de gorgojos o comején en elementos de madera.

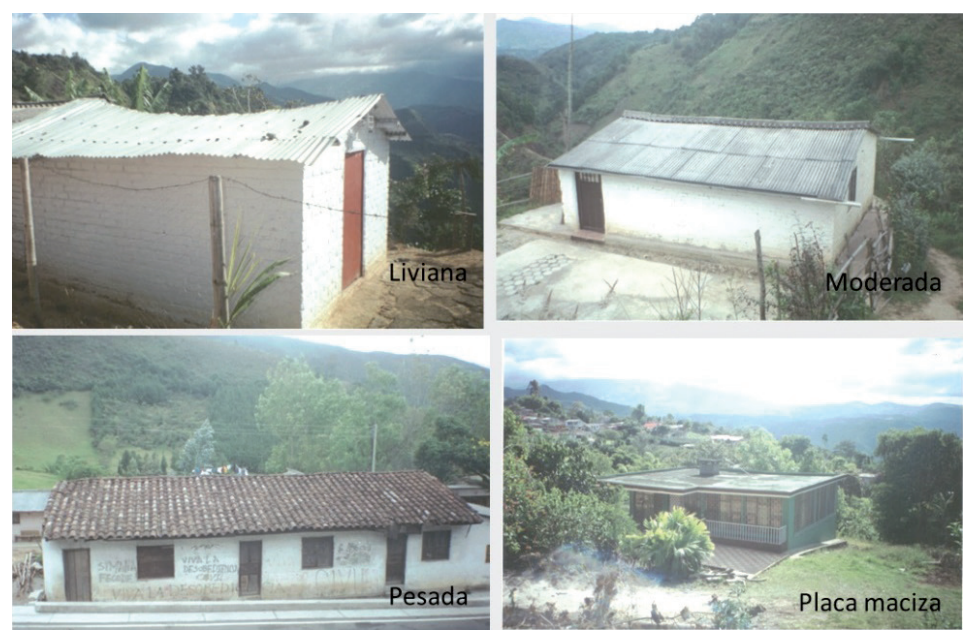

FIGURA 5. Clases de cubierta observadas en la zona de influencia del volcán Galeras. 
El estado regular se califica por la ausencia de una o varias de las condiciones del estado bueno; es decir presencia de algunas fisuras que no implican daño estructural, la no existencia de un buen acople entre los elementos estructurales o juntas, algunos indicios de humedades, alteraciones en elementos de concreto o metálicos o evidencias no muy marcadas de ataques en los elementos de madera. Para el mal estado se evidencian marcadas grietas o deformaciones en los elementos estructurales, pérdida de revestimiento en elementos de concreto, corrosión en elementos metálicos, presencia de elementos de madera podridos o muy afectado por ataques de comején o gorgojos, existen elementos desacoplados que no permiten un buen comportamiento de respuesta estructural.

La madera es un material degradable que sufre una disminución gradual de sus propiedades mecánicas, y que tan solo la hace aprovechable hasta que cumple con su vida útil. Una larga exposición de la madera a la atmósfera produce cambios en la celulosa y de acuerdo con estudios en vigas, se ha observado que el porcentaje de celulosa disminuye constantemente mientras que la lignina se mantiene casi constante. Según el manual del Ingeniero Mecánico (Marks, 1982), estas variaciones se reflejan en pérdidas de resistencia (FIGURA 6). Teniendo en cuenta este comportamiento, análogamente se asume una reducción de la resistencia a la flexión y la compresión de los elementos en madera con la edad.

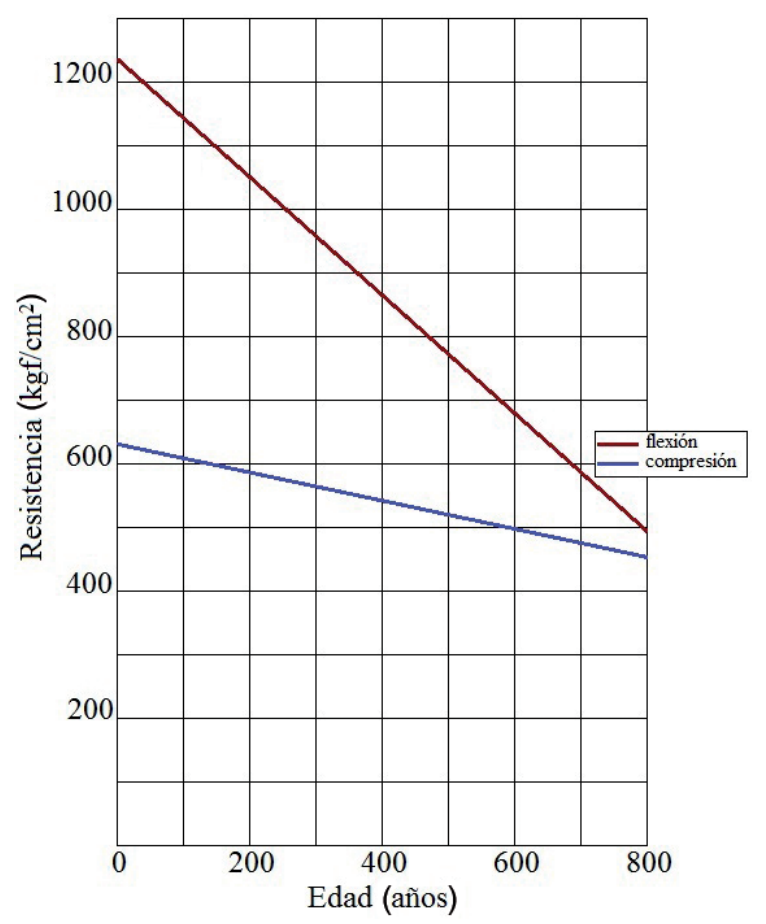

FIGURA 6. Reducción de la resistencia de la madera con los años (Tomado de Marks, 1982).
Las cubiertas tipo se determinaron teniendo en cuenta las dimensiones más comunes, la configuración y la clase de materiales utilizados. Las luces (distancias entre apoyos) y ángulos de inclinación se tomaron de observaciones directas en campo durante la etapa de inventario. En las FIGURAS 7, 8 y 9 se muestran algunos diagramas de ejemplos de estructuras de cubierta típicos observados en la zona de influencia del volcán Galeras, siendo el eucalipto la madera más comúnmente utilizada.

Según el Manual de Diseño para Maderas del Grupo Andino (PADT-REFORT, 1984), la madera puede clasificarse en grupos estructurales de acuerdo con su densidad y que permite establecer su resistencia; para el caso del eucalipto, la densidad es de 0,55 $\mathrm{g} / \mathrm{cm}^{3}$ que la cataloga en el grupo de maderas de menor resistencia. La estructura de cubierta pesada característica es la armadura tipo "Montante Maestro con diagonales o sin diagonales" o la armadura tipo "Diente de Sierra" en madera con uniones de sus elementos cuerda-cuerda, cuerda-diagonal, pendolóncuerda, etc., mediante la utilización de clavos. En cubiertas liviana-moderadas se observan también cerchas metálicas tipo "Warren", "Pratt", "Howe de cuerdas paralelas", "diente de sierra". En las FIGURAS $10,11,12$ y 13 se muestran las armaduras típicas en la zona de influencia de Galeras. La construcción puede llevarse a cabo de manera formal e informal que fundamentalmente se diferencia por la cantidad de trabajo, disponibilidad de materiales, factores climáticos, geográficos, tecnológicos, culturales y socioeconómicos. En el sector rural el método típico de construcción es el sistema informal o vernacular, dado que demanda menores costos y es ejecutada por constructores empíricos. El sistema informal utiliza métodos tradicionales y materiales de construcción con muy poca o ninguna transformación, es decir, madera rolliza, caña, paja, etc., siendo ejecutados en el lugar de la obra. El sistema informal hace que gran parte de las estructuras sean deficientes en sistemas técnico constructivos y por lo tanto incide en su resistencia y durabilidad. Otro tema considerado son los aspectos morfológicos en la estructura como son las cubiertas a una agua (tipo cobertizo), dos aguas o cuatro aguas (tipo pabellón o tipo con faldones), que se traduce en la complejidad de la estructura de soporte. En la FIGURA 9 se indican algunas morfologías típicas de cubiertas de la zona de influencia del volcán Galeras de acuerdo con el escurrimiento de aguas. 


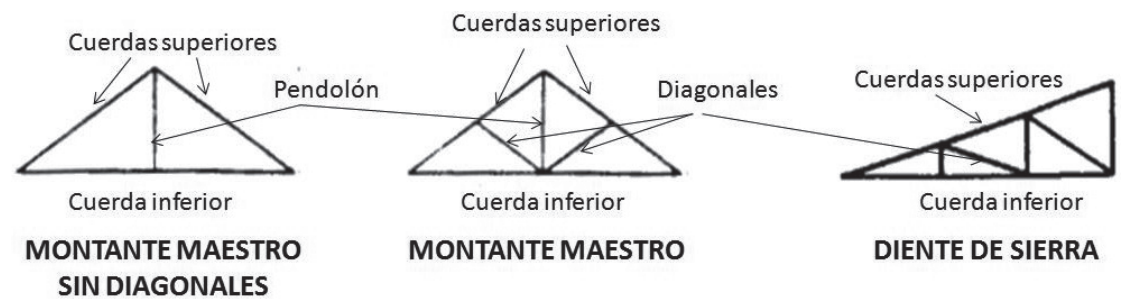

FIGURA 7. Armaduras de madera típicas en la zona de influencia de Galeras para cubiertas liviana-moderada y pesada (Tomado de Torres, 2001).

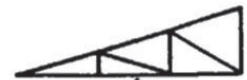

DIENTE DE SIERRA

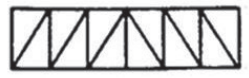

HOWE

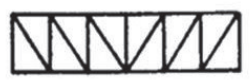

PRATT

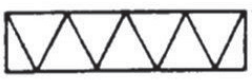

WARREN

FIGURA 8. Armaduras típicas metálicas en la zona de influencia de Galeras para cubiertas liviana-moderada (Tomado de Torres, 2001).

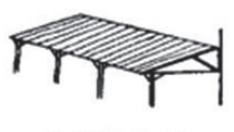

COBERTIZO

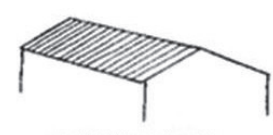

A DOS AGUAS

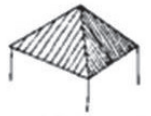

DE PABELLÓN

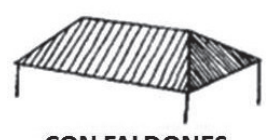

CON FALDONES

FIGURA 9. Morfologías típicas de estructuras de cubierta pesada y liviana-moderada según el escurrimiento en la zona de influencia de Galeras (Tomado de Torres, 2001).

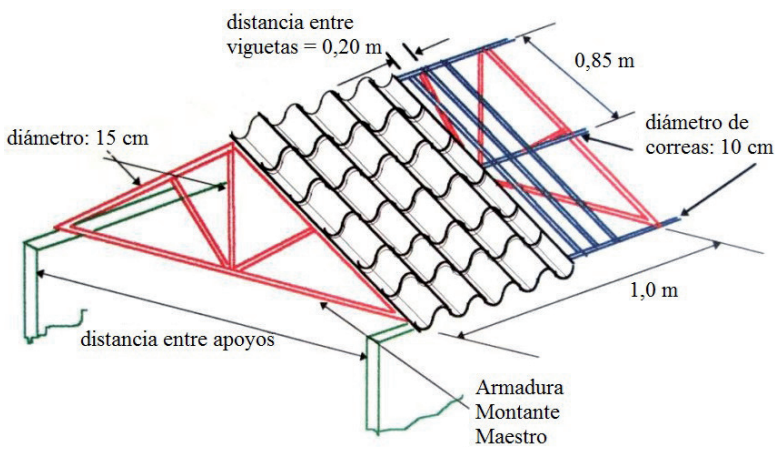

FIGURA 10. Ejemplo de una estructura de cubierta pesada observada en la zona de influencia del volcán Galeras. Los elementos que cubren la estructura corresponden a teja de barro (Tomado de Torres, 2001).

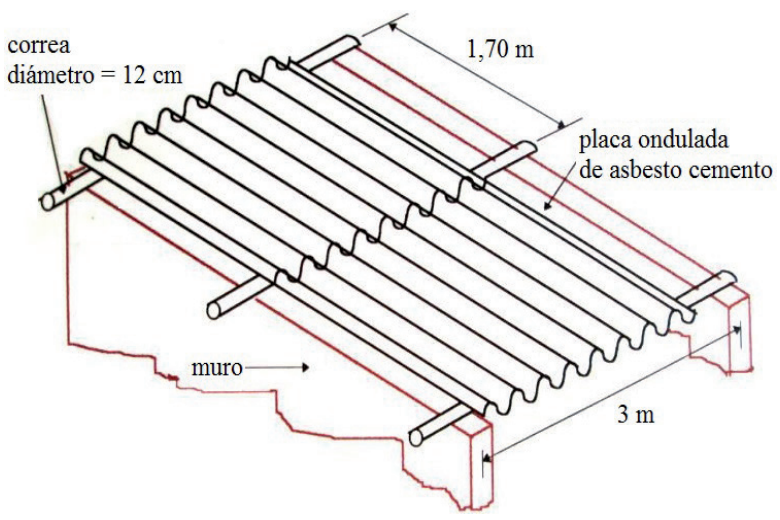

FIGURA 11. Ejemplo de una estructura de cubierta livianamoderada observada en la zona de influencia del volcán Galeras. Los elementos que cubren la estructura corresponden a teja de asbesto-cemento (Tomado de Torres, 2001).

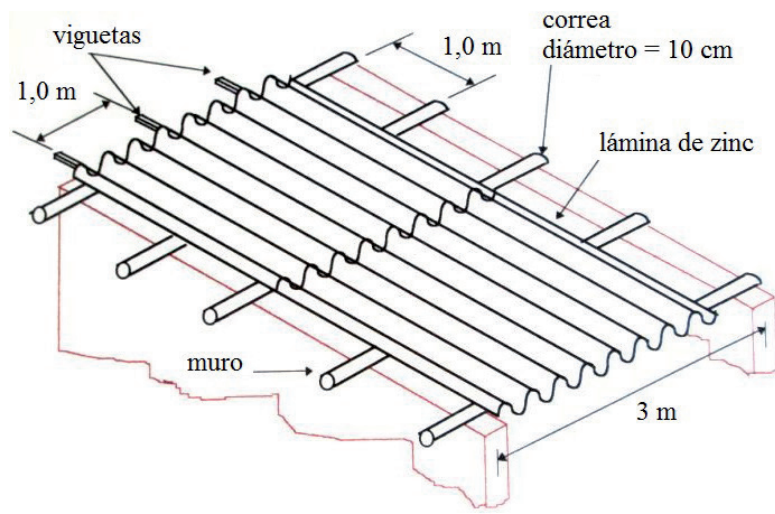

FIGURA 12. Ejemplo de una estructura de cubierta livianamoderada observada en la zona de influencia del volcán Galeras. Los elementos que cubren la estructura corresponden a lámina de zinc corrugada (Tomado de Torres, 2001).

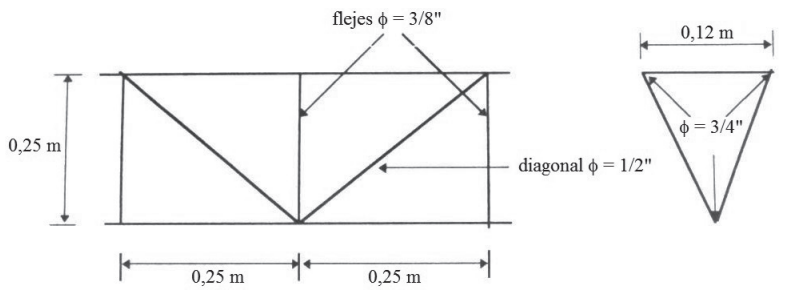

FIGURA 13. Ejemplo de una cercha que soporta una cubierta liviana-moderada conformada por placa ondulada de asbestocemento observada en la zona de influencia de Galeras (Tomado de Torres, 2001).

En la TABLA 1 se muestran las cargas de colapso en condiciones de buen estado y con inclinaciones no mayores a $20^{\circ}$, y en la FIGURA 14 se indican las 
correspondientes funciones de fragilidad para cinco clases de cubiertas identificadas en la zona de estudio con base en la estimación de las probabilidades de daño.

TABLA 1. Cargas de colapso para algunos techos en viviendas de la zona de influencia del volcán Galeras (Tomado de INGEOMINAS, 1998).

\begin{tabular}{|c|c|c|c|c|}
\hline \multirow{2}{*}{$\begin{array}{l}\text { Clase de } \\
\text { Cubierta }\end{array}$} & \multirow[t]{2}{*}{ Análisis por } & \multicolumn{3}{|c|}{$\begin{array}{c}\text { Cargas de colapso } \\
(\mathrm{kPa})\end{array}$} \\
\hline & & $Q_{\min }$ & $Q_{\text {mean }}$ & $Q_{\max }$ \\
\hline \multirow{4}{*}{ Liviana } & Flexión en la cubierta & 0,28 & 0,31 & 0,32 \\
\hline & $\begin{array}{l}\text { Módulo de sección } \\
\text { transversal en la correa }\end{array}$ & 0,65 & 0,88 & 1,38 \\
\hline & Flexión en la correa & 0,31 & 0,61 & 1,44 \\
\hline & Cortante en la correa & 1,65 & 2,74 & 4,02 \\
\hline \multirow{4}{*}{ Moderada } & Flexión en la cubierta & 0,55 & $\mathbf{0 , 5 5}$ & 0,55 \\
\hline & $\begin{array}{l}\text { Módulo de sección } \\
\text { transversal en la correa }\end{array}$ & 0,28 & 1,35 & 1,56 \\
\hline & Flexión en la correa & 0,14 & 0,97 & 1,71 \\
\hline & Cortante en la correa & 1,91 & 2,98 & 3,91 \\
\hline \multirow{3}{*}{ Pesada } & $\begin{array}{l}\text { Módulo de sección } \\
\text { transversal en la correa }\end{array}$ & 0,21 & 1,61 & 5,01 \\
\hline & Flexión en la correa & 0,71 & 1,72 & 6,06 \\
\hline & Cortante en la correa & 1,31 & 2,82 & 7,16 \\
\hline Losa Maciza & Flexión en la placa & 3,16 & 5,33 & 12,11 \\
\hline $\begin{array}{c}\text { Losa } \\
\text { Aligerada }\end{array}$ & Flexión en la placa & 2,33 & $\mathbf{5 , 5 2}$ & 11,91 \\
\hline
\end{tabular}

Nota: en negrilla se destacan los valores críticos tomados en consideración como cargas de colapso a partir de los valores promedio. Los valores mínimo y máximo son referencia para la dispersión observada.

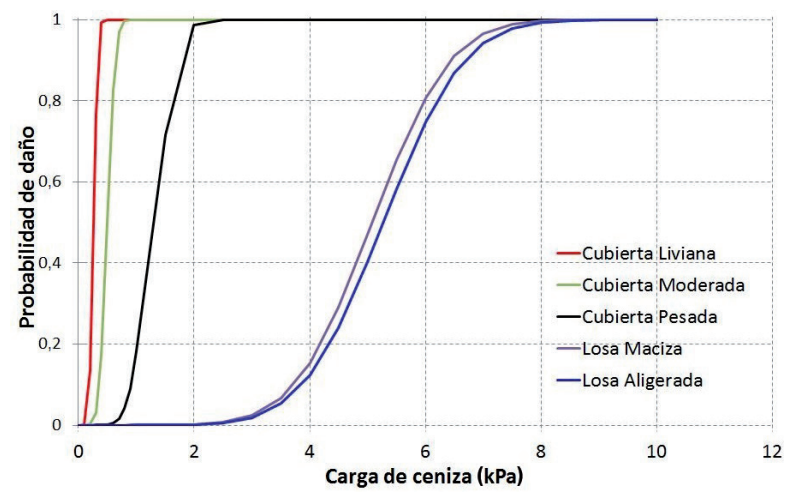

FIGURA 14. Probabilidades de daño para diferentes cargas de ceniza en cinco clases de cubiertas identificadas en la zona de influencia del volcán Galeras.

Torres (2001) hace una revaluación de las cargas de colapso establecidas por INGEOMINAS (1998), teniendo en cuenta que la resistencia de los elementos había sido afectada por unos "factores de seguridad" que en últimas diezmaban las cargas de colapso, especialmente para las cubiertas liviana, moderada y pesada. En la TABLA 2 se muestran las cargas de colapso promedio revaluadas para estas tipologías en condiciones de buen estado, con distancias entre apoyos hasta $6 \mathrm{~m}$ y en la FIGURA 15, las correspondientes probabilidades de daño. Spence et al. (2005) realizaron una propuesta de clasificación de los tipos de cubiertas europeas y hacen una estimación de la resistencia a la caída de ceniza estableciendo la fragilidad estructural de los techos de las edificaciones sobre la base de estudios analíticos, ensayos de laboratorio y análisis de daños observados. En la TABLA 3 se muestra las cargas de colapso y en la FIGURA 16 las curvas de fragilidad para cada uno de estos tipos europeos.

TABLA 2. Cargas de colapso para algunos techos en viviendas de la zona de influencia del volcán Galeras (Torres, 2001)

\begin{tabular}{|c|c|c|c|}
\hline $\begin{array}{l}\text { Clase de } \\
\text { Cubierta }\end{array}$ & Descripción & $\begin{array}{c}\text { Carga de } \\
\text { colapso, } \\
Q_{\text {mean }} \\
\end{array}$ & $\begin{array}{c}\text { Espesor } \\
\text { de ceniza } \\
\text { equivalente }\end{array}$ \\
\hline Liviana & $\begin{array}{l}\text { Techos que tienen como } \\
\text { material de cobertura hojas } \\
\text { de zinc o aluminio y hasta } \\
\text { cartón o similares como } \\
\text { marquesinas en marcolita } \\
\text { u otro material acrílico } \\
\text { o vidrio. La estructura } \\
\text { de cubierta puede ser en } \\
\text { madera o metálica. }\end{array}$ & $1,0 \mathrm{kPa}$ & $8,2 \mathrm{~cm}$ \\
\hline Moderada & $\begin{array}{l}\text { Techos que tienen como } \\
\text { material de cobertura tejas } \\
\text { de asbesto-cemento o } \\
\text { láminas predeterminadas } \\
\text { en ciertas cerámicas. La } \\
\text { estructura de cubierta } \\
\text { puede ser en madera o } \\
\text { metálica. }\end{array}$ & $1,3 \mathrm{kPa}$ & $10,6 \mathrm{~cm}$ \\
\hline Pesada & $\begin{array}{l}\text { Techos que tienen como } \\
\text { material de cobertura } \\
\text { tejones o tejas de barro. La } \\
\text { estructura que soporta la } \\
\text { cubierta usualmente es en } \\
\text { madera. }\end{array}$ & $2,5 \mathrm{kPa}$ & $20,4 \mathrm{~cm}$ \\
\hline $\begin{array}{l}\text { Losa } \\
\text { Maciza }\end{array}$ & $\begin{array}{l}\text { Placa de concreto armado } \\
\text { o bloque macizo acuñado } \\
\text { a compresión la cual va } \\
\text { soportada directamente } \\
\text { sobre muros o sistemas de } \\
\text { pórticos. }\end{array}$ & $5,5 \mathrm{kPa}$ & $44,9 \mathrm{~cm}$ \\
\hline $\begin{array}{l}\text { Losa } \\
\text { Aligerada }\end{array}$ & $\begin{array}{l}\text { Placa aligerada con nervios } \\
\text { en concreto reforzado } \\
\text { soportadas directamente } \\
\text { sobre muros o sistemas de } \\
\text { pórticos }\end{array}$ & $5,8 \mathrm{kPa}$ & $47,3 \mathrm{~cm}$ \\
\hline
\end{tabular}

Nota: los espesores equivalentes de ceniza se estimaron con un peso específico de $1250 \mathrm{~kg} / \mathrm{m}^{3}$ según Tilling (1993) para ceniza húmeda. 


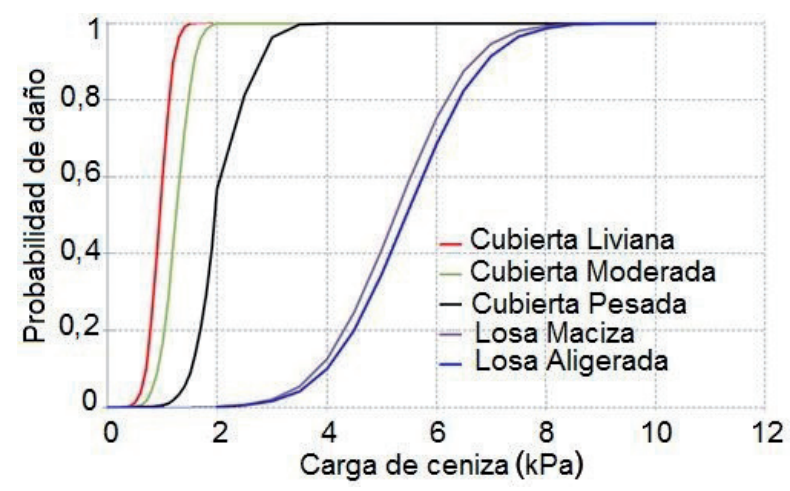

FIGURA 15. Probabilidades de daño para diferentes cargas de ceniza en cinco clases de cubiertas identificadas en la zona de influencia del volcán Galeras después de un proceso de revaluación.

TABLA 3. Cargas de colapso para algunos techos europeos (Tomado de Spence et al., 2005).

\begin{tabular}{|c|c|c|c|}
\hline $\begin{array}{l}\text { Clase de } \\
\text { Cubierta }\end{array}$ & Descripción & $\begin{array}{c}\text { Rango de } \\
\text { carga diseño } \\
\text { típico }\end{array}$ & $\begin{array}{c}\text { Carga } \\
\text { de } \\
\text { colapso, } \\
Q_{\text {mean }}\end{array}$ \\
\hline Débil (WE) & $\begin{array}{l}\text { Cubiertas de hojas } \\
\text { o tejas viejas o en } \\
\text { mal estado; techo } \\
\text { abovedado } \\
\text { mampostería }\end{array}$ & $\begin{array}{l}\text { Con código } \\
\text { pre-diseño o } \\
\text { sin código de } \\
\text { diseño }\end{array}$ & $2 \mathrm{kPa}$ \\
\hline $\begin{array}{l}\text { Medianamente } \\
\text { débil (MW) }\end{array}$ & $\begin{array}{l}\text { Hoja de techo en } \\
\text { madera de calidad } \\
\text { media; techado de } \\
\text { calidad media o } \\
\text { buena sobre vigas de } \\
\text { madera o armaduras; } \\
\text { acero o viguetas de } \\
\text { hormigón armado } \\
\text { prefabricado } \\
\text { azotea. }\end{array}$ & $1-2 \mathrm{kPa}$ & $3 \mathrm{kPa}$ \\
\hline $\begin{array}{l}\text { Medianamente } \\
\text { fuerte (MS) }\end{array}$ & $\begin{array}{l}\text { Cubierta en placa de } \\
\text { hormigón armado } \\
\text { de calidad buena; } \\
\text { techo inclinado de } \\
\text { hormigón armado. } \\
\text { Techo de hoja sobre } \\
\text { vigas de madera o } \\
\text { cerchas en buena } \\
\text { calidad y condición } \\
\text { diseñado para zonas } \\
\text { de ciclones }\end{array}$ & $2-3 \mathrm{kPa}$ & $4,5 \mathrm{kPa}$ \\
\hline Fuerte (ST) & $\begin{array}{l}\text { Cubierta en placa de } \\
\text { hormigón armado } \\
\text { diseñado para } \\
\text { acceso, construcción } \\
\text { de buena calidad y } \\
\text { resistente con menos } \\
\text { de } 20 \text { años }\end{array}$ & $>3 \mathrm{kPa}$ & $7 \mathrm{kPa}$ \\
\hline
\end{tabular}

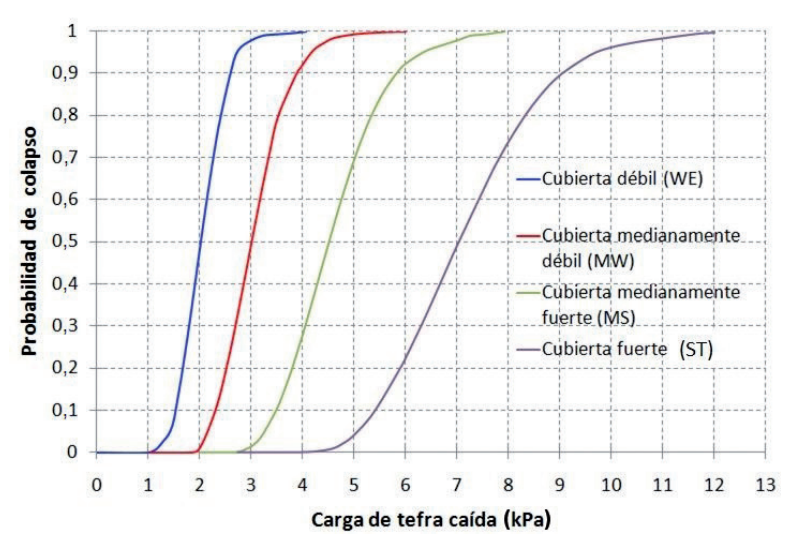

FIGURA 16. Probabilidades de daño para diferentes cargas de ceniza en cuatro clases de cubiertas europeas (Spence et al., 2005).

En la FIGURA 17 se consolida las curvas de probabilidad de daño de las cubiertas tipo de Galeras y de territorios europeos. Estableciendo una comparación de los resultados de las resistencias de las tipologías de cubiertas en la zona de influencia de Galeras estimados por Torres (2001), con las tipologías de cubiertas de territorios europeos estimadas por Spence et al. (2005), se observa en general un rango con menor resistencia, no obstante los valores encontrados son del mismo orden pese a las incertidumbres en la resistencia de algunos materiales, en las dimensiones estructurales, en la unión y acople de los elementos y formas constructivas.

Considerando un peso específico de ceniza húmeda de $1250 \mathrm{~kg} / \mathrm{m}^{3}$ (Tilling, 1993), en los resultados de Spence et al. (2005) la cubierta WE acumularía $16,3 \mathrm{~cm}$, la MW uno $24,5 \mathrm{~cm}$, la MS 36,7 cm y la ST unos $57 \mathrm{~cm}$. En la zona de influencia de Galeras, el espectro de tipologías se amplió teniendo en cuenta el estado de la cubierta según su edad y mantenimiento que se catalogó como bueno, regular o malo y la longitud entre apoyos de la cubierta considerando luces cortas (<6 m), medianas (6 a $15 \mathrm{~m})$ y largas (>15 m).

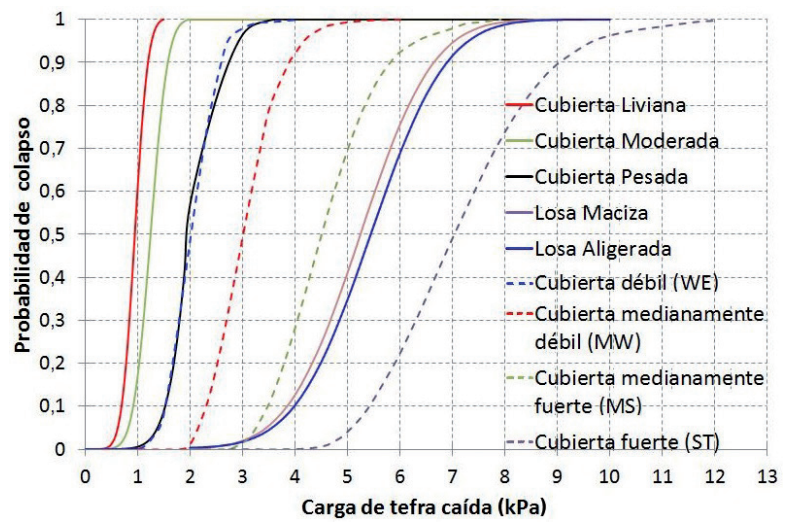

FIGURA 17. Probabilidades de daño para diferentes cargas de ceniza en los tipos de cubiertas de la zona de Galeras y de algunas regiones europeas establecidas por Torres (2001) y Spence et al. (2005) respectivamente. 
En 2012, el SGC junto con la Unidad Nacional para la Gestión del Riesgo de Desastres UNGRD - Proceso Galeras, dentro del ejercicio llevado a cabo con el TAP para una Evaluación Probabilista del Riesgo Volcánico (EPR) (SGC-UNGRD, 2012), retoma las tipologías de cubiertas definidas por INGEOMINAS (1998), estableciendo 27 tipos de techos sobre un inventario de 19155 edificaciones (Torres et al., 2012). Estas tipologías se hicieron considerando la clase, el estado y la longitud entre apoyos de la cubierta. Como resultado se obtuvo tres clases de cubiertas: Liviana-Moderada, LM (7387 edificaciones), Pesada, P (10207), Losas, L (1561). Tres condiciones de estado de acuerdo a su edad y condición de mantenimiento: Bueno, B (6503); Regular, R (11537) y Malo, M (1115). Tres rangos de distancia entre apoyos: luz corta $(<6 \mathrm{~m})$, C (13902 edificaciones); luz media (6-15m), M (5172) y luz larga (>15 m), L (81). La cubierta LM corresponde a los techos que tienen hojas de zinc, aluminio, cartón o similares como marquesinas en marcolita u otro material acrílico o vidrio y aquellas que tienen tejas de asbesto-cemento o láminas predeterminadas en ciertas cerámicas. La estructura de cubierta puede ser en madera o metálica. La cubierta $\mathrm{P}$ es para techos que tienen tejones o tejas de barro; la estructura que soporta la cubierta usualmente es en madera. La cubierta $\mathrm{L}$ puede ser Losas macizas o aligeradas soportadas directamente sobre muros o sistemas de pórticos.

Como la RMD de un elemento estructural individual no es la misma que la de toda la edificación, es necesario establecer el nivel del comportamiento global por lo que adicional a los tipos de techos, se consideraron edificaciones bajas (hasta dos pisos), B (19125) y edificaciones intermedias (de dos a cuatro pisos), I (30). En la FIGURA 18 se muestra un inventario por tipos de las 19155 edificaciones. Finalmente para el TAP se establecieron 30 diferentes tipologías de cubiertaedificación de 54 posibles, combinando la clase de cubierta, estado, la luz entre apoyos de la cubierta y la altura de las edificaciones (FIGURA 19).

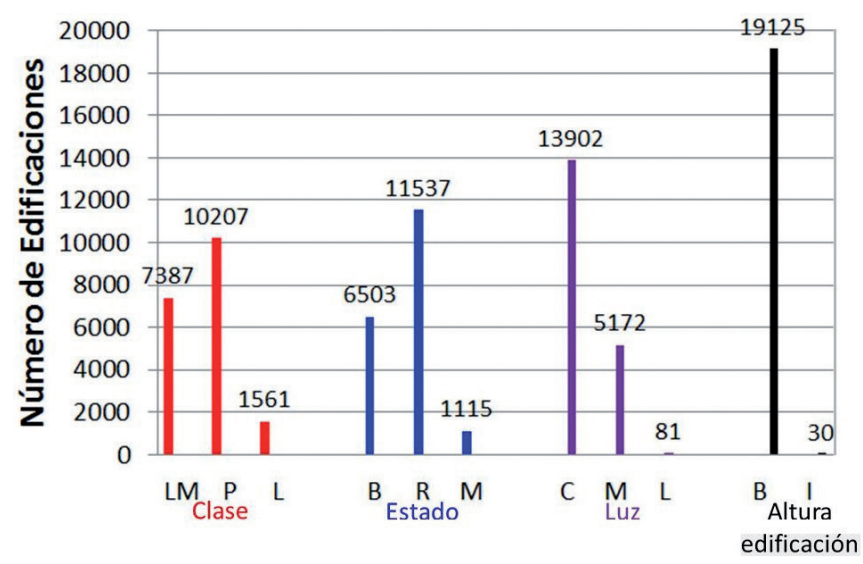

FIGURA 18. Número de edificaciones conforme a su clase de cubierta, estado, luz entre apoyos y la altura de las construcciones (Tomado de SGC-UNGRD, 2012).

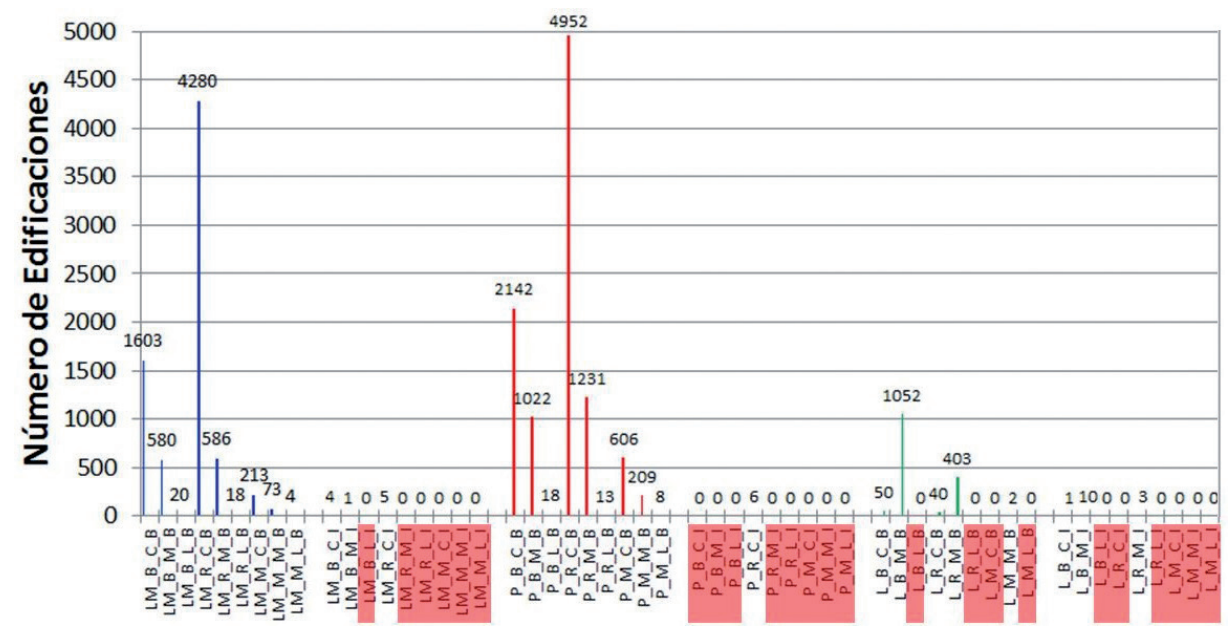

FIGURA 19. Tipologías de cubierta-edificación de las 19155 edificaciones de la zona de Galeras. Las tipologías dispuestas en las abscisas que se resaltan con rojo no están presentes en la muestra considerada (Tomado de SGC-UNGRD, 2012). 
En el TAP de 2012, para la zona de influencia del volcán Galeras, en principio se tomaron las cargas de colapso establecidas por INGEOMINAS (1998; Torres, 2001), en atención a las cargas límite de fallamiento conforme al tipo y estado de la cubierta y la distancia entre apoyos, y después de una revaluación, se modificaron algunos valores para las clases de cubiertas Liviana-Moderada (LM) teniendo en cuenta experiencias de otros estudios relacionados con cargas de colapso en cubiertas (Spence et al., 2005; Zuccaro et al., 2008).

Se asignó el mismo porcentaje dado por los estudios de Spence et al. (2005) para estimar la desviación de la carga de colapso $Q_{d e v}=20 \% Q_{\text {mean }}$. El equivalente en espesor de capa de ceniza en condiciones húmedas se obtuvo considerando un peso específico de $1250 \mathrm{kgf} /$ $\mathrm{m}^{3}$ (Tilling, 1993). En la FIGURA 20 y la TABLA 4 se muestran las cargas medias de colapso y los espesores de ceniza equivalentes para las diferentes tipologías de cubiertas y en la FIGURA 21 se grafican las probabilidades de daño correspondientes.

TABLA 4. Cargas medias de colapso y espesores de ceniza equivalentes para las diferentes tipologías de cubiertas de la zona de influencia de Galeras definidas en el TAP (SGCUNGRD, 2012).

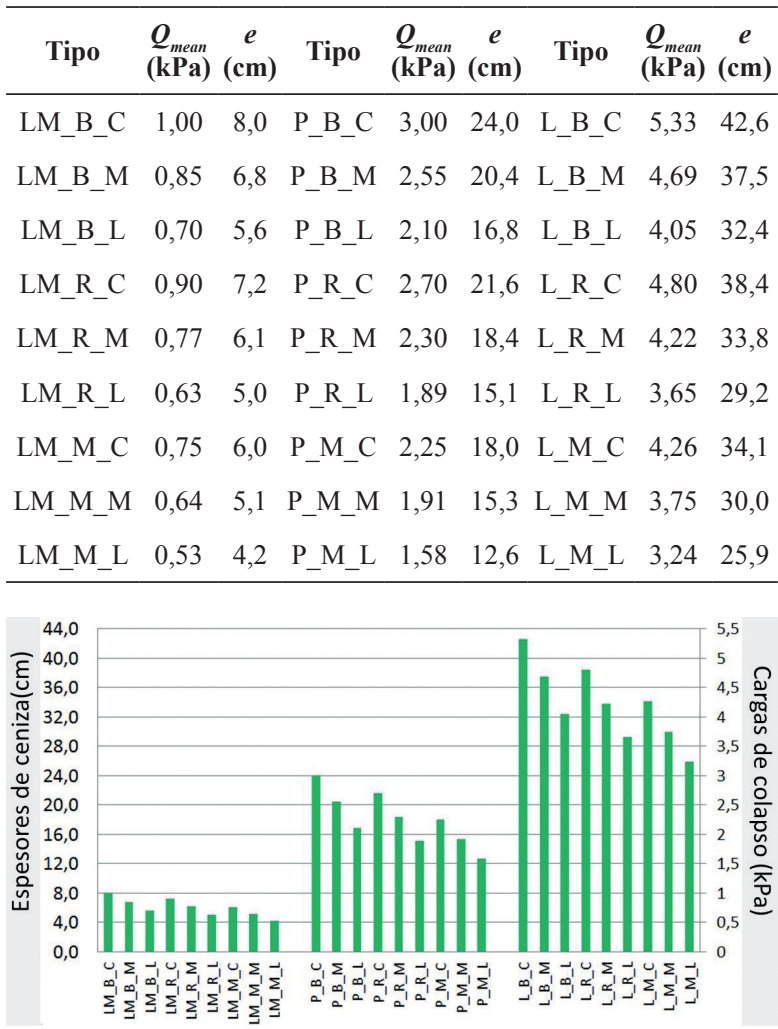

FIGURA 20. Cargas medias de colapso y los espesores de ceniza equivalentes para las diferentes tipologías de cubiertas para la zona de influencia de Galeras (Tomado de SGCUNGRD, 2012).

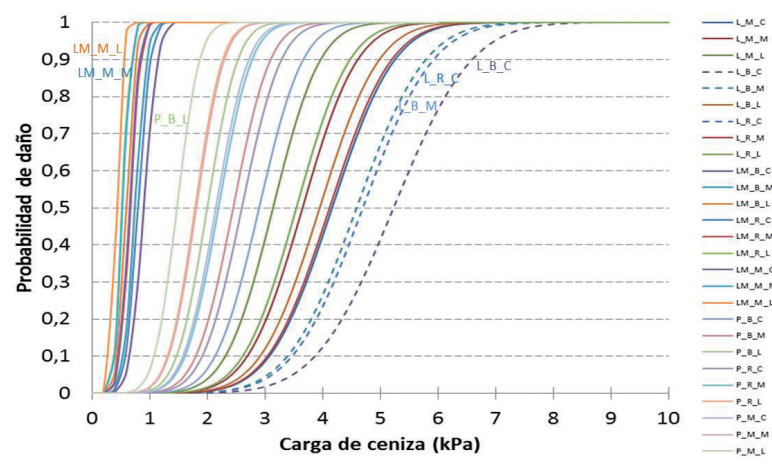

FIGURA 21. Curvas de fragilidad para cubiertas del área de Galeras ante caída de cenizas.

\section{RELACIÓN MEDIA DE DAÑO DE LA VIVIENDA ANTE CAÍDA DE CENIZAS}

Para el ejercicio del TAP (SGC-UNGRD, 2012) se consideró fundamentalmente una tipología básica de estructura para las edificaciones suponiendo muros cargueros, y se tomaron edificaciones bajas (hasta de dos pisos) y edificaciones intermedias (tres y cuatro pisos). En principio se estimaron unos valores de relaciones porcentuales de daño de la cubierta respecto a la edificación, $D_{\max }$, atendiendo solamente a nuestro criterio y experiencia, tal como se muestran en la TABLA 5 y la FIGURA 22.

TABLA 5. Relaciones porcentuales de daño de la cubierta respecto a la edificación, $D_{\max }$.

\begin{tabular}{cccccccc}
\hline Clase & Luz/Estado & BB & BI & RB & RI & MB & MI \\
\hline LM & C & 5 & 3 & 6 & 4 & 7 & 4 \\
LM & M & 7 & 4 & 8 & 5 & 9 & 5 \\
LM & L & 10 & 6 & 11 & 6 & 12 & 7 \\
P & C & 20 & 13 & 28 & 14 & 35 & 23 \\
P & M & 25 & 17 & 31 & 16 & 37 & 25 \\
P & L & 30 & 20 & 35 & 18 & 40 & 27 \\
L & C & 70 & 80 & 55 & 60 & 40 & 45 \\
L & M & 80 & 90 & 65 & 70 & 50 & 60 \\
L & L & 90 & 100 & 75 & 100 & 60 & 100 \\
\hline
\end{tabular}

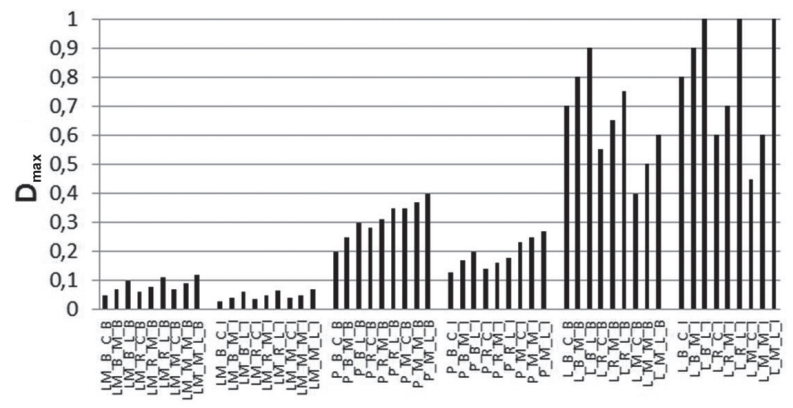

FIGURA 22. Relaciones porcentuales de daño de la cubierta respecto a la edificación, $D_{\max }$. 


\section{VULNERABILIDAD PARA CUBIERTAS ANTE CAÍDA DE CENIZAS}

Las funciones de vulnerabilidad varían en función de la clase de cubierta y del tipo de edificación. En la FIGURA 23 se muestran las funciones de daño esperadas para las diferentes tipologías de cubierta en edificaciones bajas. En general, de acuerdo con las FIGURAS 15, 20 y 21, se destaca que las Losas son las cubiertas más resistentes ante cargas de ceniza, seguida por la cubierta Pesada y la menos resistente es la cubierta Liviana-Moderada. En las cubiertas con teja el tipo de estructura de soporte desempeña un papel fundamental dentro del sistema estructural secundado por la sección de los elementos de apoyo, y finalmente por el material de soporte. Desde el punto de vista de resistencia global de la estructura tienen mayor ponderación la resistencia de la cobertura de techo y el tipo de estructura de soporte seguido por las uniones o ensambles.

Entonces, por las características constructivas y estructurales las cubiertas Liviana-Moderada son más vulnerables que las Pesadas debido a la conformación estructural, dado que la cubierta Pesada presenta mayor cantidad de elementos que se interconectan entre sí como son las armaduras o cerchas, correas y elementos de apoyo (usualmente en madera) para las tejas de barro que se disponen a distancias más cortas, en comparación con las cubiertas Liviana-Moderada, que tienen distancias más largas entre apoyos debido al tamaño de la teja. La teja de barro junto con el elemento de apoyo conforman una estructura articulada y flexible que puede redistribuir mejor las cargas a las que puede estar sometida, a diferencia de las tejas de zinc o tejas de asbesto-cemento, que por sus características funcionales pueden cubrir luces mayores, mediante apoyos simples, con vigas o correas de espesores de menor sección por el peso de la teja, resultando más frágiles a la solicitación de las cargas impuestas por la ceniza. En el sistema de cubiertas de Losa en concreto, el elemento más importante es el sistema estructural secundado por la distancia entre apoyos y la resistencia alcanzada por el concreto (calidad).

El estado de conservación de la cubierta es un factor importante en la respuesta a las solicitaciones de sobrecarga impuestas en la estructura y en las viviendas de la zona de influencia de Galeras, es más marcado en las cubiertas que involucran elementos de madera como son las Pesadas y Liviana-Moderada, lo que implica que debe hacerse un mantenimiento más seguido en el tiempo en comparación con las Losas. En el caso de colapso total, las Losas en buen estado conllevan a un mayor daño sobre toda la edificación, no obstante, resulta menos probable que se logren mayores deposiciones de ceniza acumulada que conlleven a solicitaciones de carga más grandes, y por tanto este tipo de cubiertas resultan ventajosas desde el punto de vista de resistencia.

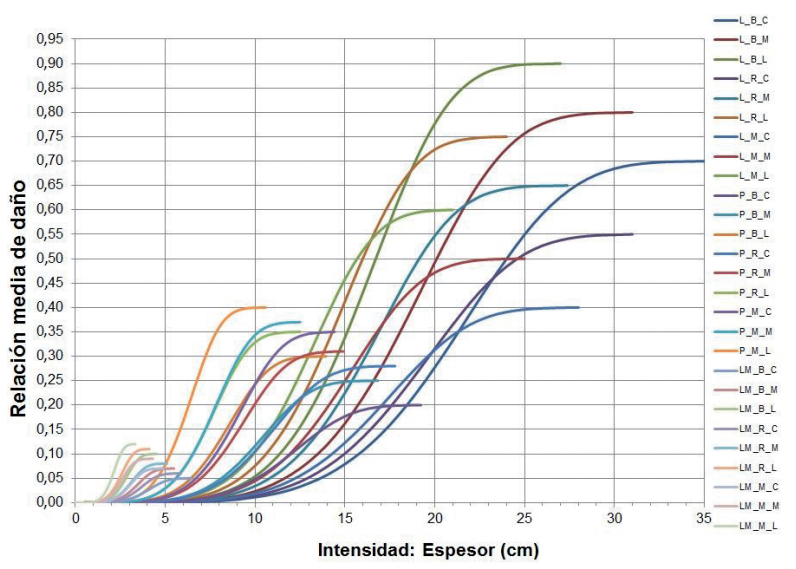

FIGURA 23. Funciones de daño esperadas según los valores de espesores de cenizas para las diferentes tipologías de cubierta en edificaciones bajas en la zona de influencia de Galeras.

En relación del daño de la cubierta con respecto a la edificación, como se ilustra en la FIGURA 22, se destaca que las cubiertas Liviana-Moderada tienen menor implicación global sobre la estructura en contraste con las Losas, dado que el colapso de las últimas, que pueden resistir mayor sobrecarga aunado a su propio peso, pueden colapsar toda la edificación, además, las Losas facilitan que se pueda llevar a cabo una limpieza de la ceniza que se va depositando, evitando que se tengan acumulaciones considerables de ceniza que afecte la estructura.

La inexistencia de mecanismos de limpieza o la falta de una pendiente adecuada son factores que amplifican la vulnerabilidad de las cubiertas Liviana-Moderada. Aunque el efecto de la pendiente de la edificación pueda resultar no muy eficiente quizás debido a las condiciones de humedad de la ceniza con relación al ángulo de reposo, la falta de una pendiente adecuada y la falta de mecanismos de limpieza hacen que las cubiertas Liviana-Moderada y Pesada sean más susceptibles a colapso por la acumulación de espesores de ceniza.

\section{CONCLUSIONES Y RECOMENDACIONES}

Se ha elaborado una metodología para evaluación de la vulnerabilidad física de cubiertas de viviendas ante la sobrecarga por caída de cenizas volcánicas, teniendo 
en cuenta las experiencias relacionadas con la amenaza volcánica por productos de caídas piroclásticas en la zona de influencia del volcán Galeras. Los inventarios de viviendas ubicadas en esta zona y estudios conducentes a la evaluación de las resistencias de algunas tipologías estructurales presentes en 1998, 2001 y 2012, fueron realizados por el SGC.

La evaluación de la vulnerabilidad ante caída de cenizas de Galeras permite estimar el grado de daño probable de una edificación según el tipo de cubierta y la solicitación a la que puede estar sometida y sirve como referente para estudios en otras regiones volcánicas; sin embargo, es importante establecer las tipologías propias de cada zona.

Aunque las cubiertas Liviana-Moderada son más vulnerables a las cargas de cenizas, las edificaciones son menos susceptibles de afectación global, contrario a las cubiertas Pesadas y las Losas.

A fin de reducir los daños ante caída de cenizas, desde el diseño conviene formular pendientes adecuadas a las cubiertas o proveer mecanismos de limpieza mecánicos o accesos para permitir la remoción de la ceniza que se vaya acumulando y evitar la sobrecarga que pueda ocasionar el daño.

Este compendio de experiencias en la evaluación de la vulnerabilidad de cubiertas en la zona de Galeras constituye un aporte a consideraciones que deberían tenerse en las cubiertas de edificaciones ubicadas en zonas aledañas a volcanes activos y que podrían ser la base para la incorporación de estas cargas en las normas de diseño y construcción para cubiertas resistentes ante sobrecargas de ceniza.

Es indispensable invertir mayores esfuerzos en la evaluación de la respuesta estructural, el mecanismo de falla y el grado de daño de las cubiertas y de la edificación, teniendo en cuenta tratamientos analíticos y de simulación, ensayos de laboratorio y análisis sobre experiencias de daños observados que tengan en cuenta el estado de cada uno de los componentes a fin de tener resultados validados y más ajustados a la realidad.

\section{AGRADECIMIENTOS}

Al Servicio Geológico Colombiano por apoyar y facilitar los datos para este estudio, al Proyecto de Asistencia Técnica del Banco Mundial (programa CAPRA), a la Unidad Nacional para la Gestión del Riesgo de Desastres UNGRD - Proceso Galeras, al grupo consultor
ERN-AL, al Global Facility for Disaster Reduction and Recovery (GFDRR), a los dos revisores por sus amables observaciones que hicieron posible que el manuscrito fuera más claro.

\section{REFERENCIAS}

ATC-13. 1985. Earthquake damage evaluation for California. Applied Technology Council, Report No. 13. Redwood City, USA, 492p.

Calvache, M. 1990. Geology and volcanology of the recent evolution of Galeras volcano, Colombia. MSc. Thesis, Louisiana State University, USA. 171p.

CORPOSSO. 2009. Evaluación de la vulnerabilidad física y funcional a fenómenos volcánicos, en el área de influencia del Volcán Galeras. Convenio de Cooperación $\mathrm{N}^{\circ}$ 1005-08-12-07 entre el Fondo nacional de Calamidades y la Corporación OSSO. Pasto, 535p.

Díaz-Granados, M. 1994. Elementos probabilísticos del riesgo. Curso de educación continuada, evaluación y mitigación de riesgos naturales. Universidad de Los Andes. Bogotá, 17p.

Dorado, L. 2013. Evaluación de vulnerabilidad de edificaciones ante caída de ceniza por eventos eruptivos del volcán Galeras en la cabecera urbana del municipio de Consacá - Departamento de Nariño. Tesis de Maestría, Desarrollo Sustentable con énfasis en Prevención y Atención de Desastres. Universidad del Valle. Cali, 88p.

ERN-AL. 2009. Vulnerabilidad de edificaciones e infraestructura. Informe Técnico ERN-CAPRA-T1-5. Evaluación de Riesgos Naturales América LatinaERN-AL.

INGEOMINAS. 1997. Memoria del mapa de amenaza volcánica del Galeras. Tercera versión. Publicación Especial de INGEOMINAS. Bogotá. 38p.

INGEOMINAS. 1998. Evaluación de la vulnerabilidad de cubiertas de viviendas expuestas a la caída de ceniza en la zona de influencia del volcán Galeras. INGEOMINAS. Pasto. 96p.

López, O. 1997. Evaluación de la resistencia estructural de las cubiertas de las edificaciones ubicadas en la zona de influencia del volcán Galeras. INGEOMINAS. Pasto, 102p. 
Marks, L. 1982. Manual del Ingeniero Mecánico. México D.F.: Ed. Baumeister Theodore. McGraw Hill.

Miranda, E. 1999. Approximate seismic lateral deformation demands in multistory buildings. Journal of Structural Engineering, 125(4): 417-425.

Ordaz, M. 2000. Metodología para la evaluación del riesgo sísmico enfocada a la gerencia de seguros por terremoto. Universidad Nacional Autónoma de México. México DF.

PADT-REFORT. 1984. Manual de diseño para maderas del Grupo Andino. Proyectos Andinos de Desarrollo Tecnológico en el área de los Recursos Forestales. Junta del Acuerdo de Cartagena. Lima, Perú, 485p.

SGC-UNGRD. 2012. Análisis probabilista de riesgo volcánico para el volcán Galeras - Colombia. Servicio Geológico Colombiano SGC y Unidad Nacional para la Gestión del Riesgo de Desastres - UNGRD. Technical Assistance Project (TAP) con fondos del Banco Mundial y el Global Facility for Disaster Reduction and Recovery (GFDRR). Asistencia Técnica del consorcio ERN-AL. Bogotá, 72p.

Spence, R., Pomonis, A., Baxter, P., Coburn, A., White, M., and Dayrit, M. 1996. Building damage caused by the Mount Pinatubo eruption of June 15, 1991. In: Newhall, C.G., and Punongbayan, R. (Eds.). Fire and Mud: Eruptions and Lahars of Mount Pinatubo, Philippines. Philippine Institute of Volcanology and Seismology, Quezon City.

Spence, R., Kelman, I., Baxter, P., Zuccaro, G., and Petrazzuoli, S. 2005. Residential building and occupant vulnerability to tephra fall. Natural Hazards and Earth System Sciences, 5: 477-494.

Tilling, R. 1993. Volcanic hazards. World Organization of Volcano Observatories (WOVO - IAVCEI). Santa Fe, New Mexico, 125p.

Torres, R. 2001. Vulnerabilidad estructural de edificaciones de uso general ante eventos eruptivos del volcán Galeras en su zona de influencia. INGEOMINAS. Pasto, 122p.

Torres, R., and Cárdenas, R. 2006. Vulnerability of normal occupation houses before the ash fall of the Galeras volcano. Fourth Conference Cities on Volcanoes, International Association of Volcanology and Chemistry of the Earth's Interior - IAVCEI. Quito, pp. 141
Torres, R., Ponce, P., y Gómez, D. 2012. Estimación de funciones de vulnerabilidad ante erupciones volcánicas. Servicio Geológico Colombiano SGC. Technical Assistance Project (TAP) con fondos del Banco Mundial y el Global Facility for Disaster Reduction and Recovery (GFDRR). Asistencia Técnica del consorcio ERN-AL. Pasto, 13p.

UNDRO. 1991. UN Office of the Disaster Relief Coordinator. Mitigating natural disasters: Phenomena, effects and options: A manual for policy makers and planners. United Nations Publication. Nueva York, 164 p.

Zuccaro, G., Cacace, F., Spence, R., and Baxter, P. 2008. Impact of explosive eruption scenarios at Vesuvius. Journal of Volcanology and Geothermal Research, 178(3): 416-453.

Trabajo recibido: noviembre 22 de 2016

Trabajo aceptado: abril 11 de 2017

Manuscrito publicado en internet: mayo 05 de 2016 